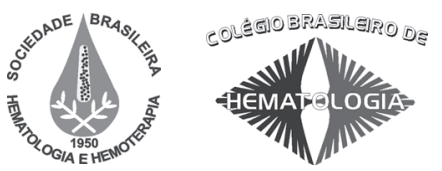

\title{
Avaliação do perfil hematológico de pacientes com leucemia linfocítica crônica (LLC-B) em um hemocentro estadual
}

\author{
Evaluation of the hematologic profile of patients with chronic lymphocytic leukemia (CLL-B) \\ at a state hemocenter
}

Romélia P. Gonçalves ${ }^{1}$

Daniel P. Maia

Richeyla K. A. Custódio

Rosângela P. G. Machado

Fernando B. Duarte 4

Lilianne B. Silva ${ }^{2}$
Tem sido dada grande importância aos avanços na biologia molecular e genética no estabelecimento de novos protocolos, bem como à descoberta de novos marcadores tumorais, úteis ao diagnóstico e tratamento precoces de várias doenças neoplásicas, inclusive a leucemia linfocítica crônica de células B (LLC-B). Para avaliação da atual significância do sistema de estadiamento clínico de Rai no prognóstico de 50 pacientes com $L L C$-B em um hemocentro estadual, bem como para comparação entre os resultados do estadiamento com valores séricos de $\mathrm{LDH}$ e CD38 ao diagnóstico, foram coletados dos prontuários dos pacientes dados referentes à contagem total de linfócitos e plaquetas, concentração de hemoglobina, e também informações quanto à presença ou ausência de linfadenopatia elou organomegalia no periodo de admissão. Quando comparados os resultados do estadiamento com aqueles de outros estudos, observou-se uma situação preocupante quanto ao percentual superior de pacientes já classificados como de alto risco ao diagnóstico. Este quadro de aparente atraso na detecção da LLC-B foi em parte atribuído à dificuldade de acesso a centros especializados elou atraso na avaliação hematológica. Além disso, observaram-se incoerências entre os valores de LDH e CD38, e entre estes e o sistema de classificação clínica de Rai. Os resultados sugerem que este sistema de classificação pode ainda ser útil como panorama geral comparativo da LLC-B entre diferentes populações, mas também enfatizam a necessidade de modelos prognósticos especificos que considerem, além dos dados clínicos e marcadores CD38 e LDH, outros indicadores mais precisos do status mutacional IgVH para prognósticos e terapias mais exatos. Rev. Bras. Hematol. Hemoter. 2009;31(4):228-234.

Palavras-chave: Perfil hematológico; leucemia linfocítica crônica; prognóstico.

\section{Introdução}

ALLC-B é uma doença de maior prevalência no idoso, com médias de idade entre 64-70, caracterizada pelo acúmulo de linfócitos B no sangue periférico, medula óssea e órgãos linfóides sólidos. ${ }^{1,2,3}$ Os sistemas clássicos de estadiamento da doença, Rai ${ }^{14}$ e Binet, ${ }^{15}$ são baseados em características clínicas e hematológicas (Tabela 1). ${ }^{3}$ Contudo, em estágios iniciais da LLC-B, nenhum dos dois sistemas distingue com precisão os pacientes que podem desenvolver a forma mais agressiva da doença em relação aos que permanecerão com a forma mais indolente. ${ }^{2}$

Estes sistemas definem estágios precoce (Rai 0, Binet A), intermediário (Rai I/II, Binet B) e avançado (Rai III/IV,

${ }^{1}$ Professora Associada de Hematologia da Universidade Federal do Ceará - Fortaleza-CE.

${ }^{2}$ Farmacêutico(a)- Bioquímico(a).

${ }^{3}$ Médica do Serviço de Hematologia da UFC/Hemoce - Fortaleza-CE.

${ }^{4}$ Médico da Maternidade Escola - Fortaleza-CE.

Universidade Federal do Ceará - Fortaleza-CE

Correspondência: Lilianne Brito da Silva

Rua Dr. José Lourenço, 1820, apto 502 - Aldeota

60115-281-Fortaleza-CE - Brasil

E-mail:liliannebrito@hotmail.com

Doi:10.1590/S1516-84842009005000064 
Tabela 1. Classificação dos cinco estágios de estadiamento segundo Rai, 1975. Hb, Hemoglobina. (adaptado de Wintrobe, 2003) $^{3}$

\begin{tabular}{cc}
\hline Rai & Características clínicas dos estágios Rai \\
\hline 0 & Linfocitose apenas $\left(>=15.000 / \mathrm{mm}^{3}\right.$ no sangue \\
ou $>40 \%$ na medula)
\end{tabular}

Binet C), com sobrevida, respectivamente: $>10$ anos, 5-7 anos e 1-3 anos. Os sistemas de estadiamento não permitem predizer a taxa de progressão da doença em pacientes em estágios iniciais nem a probabilidade de resposta ao tratamento naqueles de estágio mais avançado. ${ }^{5}$

Aproximadamente $50 \%$ dos pacientes com LLC são assintomáticos ao contato inicial e apresentam linfocitose no sangue periférico isolada e sem causa determinada. Sintomas constitucionais estão presentes em aproximadamente $15 \%$ dos pacientes ao diagnóstico, sendo suor noturno, perda de peso e fadiga mais frequentes que febre relacionável à doença. Os achados físicos mais comuns são linfadenopatia, seguida de esplenomegalia e hepatomegalia. Envolvimento extranodal de tonsilas e pele tem sido observado, enquanto envolvimentos do trato gastrointestinal, pulmão, pleura, sistema nervoso central, rins e ossos são incomuns. ${ }^{7}$

Quanto às características laboratoriais, os exames mais utilizados e úteis à tentativa de acerto diagnóstico laboratorial e prognóstico são: o hemograma completo, tempo de duplicação linfocitária, o padrão de infiltração histológico em biópsias, marcadores séricos, imunofenotipagem, análise citogenética para deteç̧ão de aberrações cromossômicas e avaliação do estado de mutação de genes da cadeia pesada da região variante de imunoglobulina $\left(\operatorname{IgV}_{\mathrm{H}}\right)$ através de marcadores específicos. ${ }^{3}$ Alguns marcadores sorológicos como $\beta 2$ microglobulina ( $\beta 2-\mathrm{MG})$, desidrogenase lática ( $\mathrm{LDH}$ ), timidina quinase (s-TK) e CD23 sérico (s-CD23) têm sido acessíveis à maioria dos serviços de onco-hematologia, embora não possam contribuir significativamente para a avaliação de prognóstico na LLC. 6,8

Uma série de estudos demonstra que a LLC-B pode ser dividida em dois subgrupos dependendo da presença (bom prognóstico) ou ausência (mau prognóstico) de mutações nos genes $\operatorname{IgV}_{\mathrm{H}}$, com um cut-off de $2 \%$ de diferença em relação ao status genético dos genes de linhagem germinativa correspondente. Além do status de mutação $\operatorname{IgV}_{\mathrm{H}}$, dois outros marcadores úteis ao prognóstico da LLCB são o CD38 e o ZAP-70. ${ }^{4}$ Alguns estudos sugerem um papel prognóstico superior de ZAP-70 em relação ao CD38 e status mutacional $\operatorname{IgV}_{\mathrm{H}}$.
CD38 foi detectado na superfície de células de uma proporção de casos de LLC-B e sua expressão foi correlacionada com algumas propriedades funcionais destas células, como a propensão de morrer por apoptose in vitro, tanto espontaneamente como depois de ligação cruzada com IgM de superfície. A expressão de CD38 é um indicador de mal prognóstico, em alguns casos relacionado também à nãomutação nos genes $\operatorname{IgV}_{\mathrm{H}}$. Embora esta última relação nem sempre tenha sido confirmada nos vários estudos, a expressão de CD38 constitui um importante e independente fator prognóstico negativo na grande maioria. ${ }^{4}$

O limite mais coerente para definição quanto à positividade de CD38 é ainda discutido, tendo sido proposto os seguintes pontos de corte: $5 \%, 7 \%, 20 \%$ e $30 \%$, este último de melhor aceite. ${ }^{4,10}$ Resultados controversos entre CD38 e ZAP-70 têm também sido observados. ${ }^{10-13}$

Desta forma, decidimos, neste trabalho, realizar uma análise comparativa entre as variáveis relativas ao estadiamento clínico segundo Rai et al. e dois marcadores tumorais então disponíveis - LDH e CD38.

\section{Casuística e Método}

Realizou-se um estudo transversal em uma população de 50 pacientes com LLC-B, escolhidos aleatoriamente dentre os casos confirmados, pertencentes ao Centro de Hematologia e Hemoterapia do Estado do Ceará (Hemoce). O diagnóstico de todos os pacientes fora confirmado, previamente ao estudo, através de avaliação clínica, imunofenotipagem e mielograma, e, quando necessário, também por biópsia óssea.

Os prontuários foram disponibilizados à medida que estes pacientes iam retornando às consultas seguintes, dentro do período de estudo (2006 a 2007). Como critério de exclusão, considerou-se apenas a ausência de dados essenciais ao estadiamento clínico segundo Rai, dando-se preferência também para aqueles pacientes com valores já disponíveis de LDH e CD38. Os dados coletados foram referentes apenas ao período inicial de diagnóstico.

Para avaliação estatística dos dados, utilizou-se os softwares SPSS versão 15 e R versão 2.8.0. Calcularam-se as frequências e médias quanto à distribuição de cada variável numérica na população. Nos gráficos, para o teste de normalidade, utilizou-se o teste Kolmogorov-Smirnov, com o cálculo do Valor-P e confirmação ou rejeição das respectivas hipóteses de normalidade quanto às distribuições.

Realizou-se também uma avaliação comparativa entre as variáveis, através de gráficos comparativos e testes de Kruskal-Wallis e da Mediana, com seus respectivos Valores$\mathrm{P}$, a fim de se confirmar ou não uma igualdade ou diferença quanto às variáveis numéricas comparadas duas-a-duas, ou entre variáveis numéricas e categóricas.

Gráficos de dispersão para correlações lineares ou exponenciais também foram feitos para todas as variáveis, calculando-se, para tanto, os coeficientes de correlação linear 
(r) e de determinação $\left(\mathrm{R}^{2}\right)$, e sugerindo-se, através de regressão linear, via teste $\mathrm{F}$, modelos ajustados para os casos em que o Valor-P encontrado sugerisse alguma significância dentre cada combinação de variáveis.

A maioria dos gráficos (22 no total), entretanto, não pôde ser adicionada nesta publicação, mas estão disponíveis a quem for de interesse via solicitação aos autores.

Este estudo foi submetido e aprovado pelo Comitê de Ética do setor de Ensino e Pesquisa do Hemoce, como parte de um estudo mais amplo acerca da avaliação de novos marcadores tumorais no prognóstico clínico e terapêutico destes pacientes, pelo mesmo grupo de pesquisa.

\section{Resultados}

Quanto à idade, observou-se uma normalidade de distribuição (Valor-P $=0.942$ ) dentre a população estudada (Gráfico 1). Teve-se no estudo uma distribuição de 4\% (n=2) de pacientes na faixa-etária de 40-50 anos, $12 \%(\mathrm{n}=6)$ entre 50-60 anos, $30 \%(\mathrm{n}=15)$ entre $60-70$ anos, $38 \%(\mathrm{n}=19)$ entre $70-80,12 \%(n=6)$ entre $80-90$ anos e apenas $4 \%(n=2)$ acima de 90 anos de idade. A média calculada para a idade foi de $69,84( \pm 10,95)$ anos. Quanto ao sexo e origem, observou-se $56 \%(\mathrm{n}=28)$ do sexo masculino e $44 \%(\mathrm{n}=22)$ de pacientes do sexo feminino, sendo $70 \%(n=35)$ da capital e $30 \%(n=15)$ do interior do estado. As variáveis do hemograma, bem como as das idades e LDH, podem ser visualizadas quanto a seus valores estatísticos na Tabela 2.

Quanto à concentração de hemoglobina, especificamente, os dados mostraram que, dos 50 pacientes, $16(32 \%)$ apresentavam anemia no momento do diagnóstico, com hemoglobina variando de 7,5 a 11,4 g/dL, tomando-se como ponto de corte 11,5 g/dL, apresentando uma distribuição normal dentre a população (Valor-P=0.982). Para a classificação Rai adotou-se o ponto de corte de $11 \mathrm{~g} / \mathrm{dL}$, segundo metodologia original de Rai et al. O valor médio da concentração de hemoglobina encontrado foi de 12,26 $( \pm 2,25) \mathrm{g} / \mathrm{dL}$.

Avaliou-se também a prevalência de trombocitopenia na população, com um valor de corte de 150.000 plaquetas/ $\mu \mathrm{L}$, obtendo-se um percentual de $44 \%(\mathrm{n}=22)$ dentre os 50 pacientes com concentração de plaquetas abaixo do normal e uma normalidade quanto à distribuição das concentrações de plaquetas dentre a população estudada (Valor-P $=0.166$ ). Para o estadiamento Rai considerou-se, entretanto, o ponto de corte de 100.000 plaquetas $/ \mu \mathrm{L}$, segundo metodologia original descrita por Rai et al. A média de concentração plaquetária foi de $190.079( \pm 104.856)$ plaquetas $/ \mu \mathrm{L}$.

Ainda, 24 pacientes (48\%) apresentaram em seus prontuários relatos de linfadenopatia quando na data de diagnóstico, enquanto em 16 (32\%) foi observada organomegalia (fígado e/ou baço).

Tomando-se como base os dados clínico-laboratoriais acima, pôde-se definir o estadiamento para os 50 pacientes deste estudo, seguindo-se os critérios já mencionados para

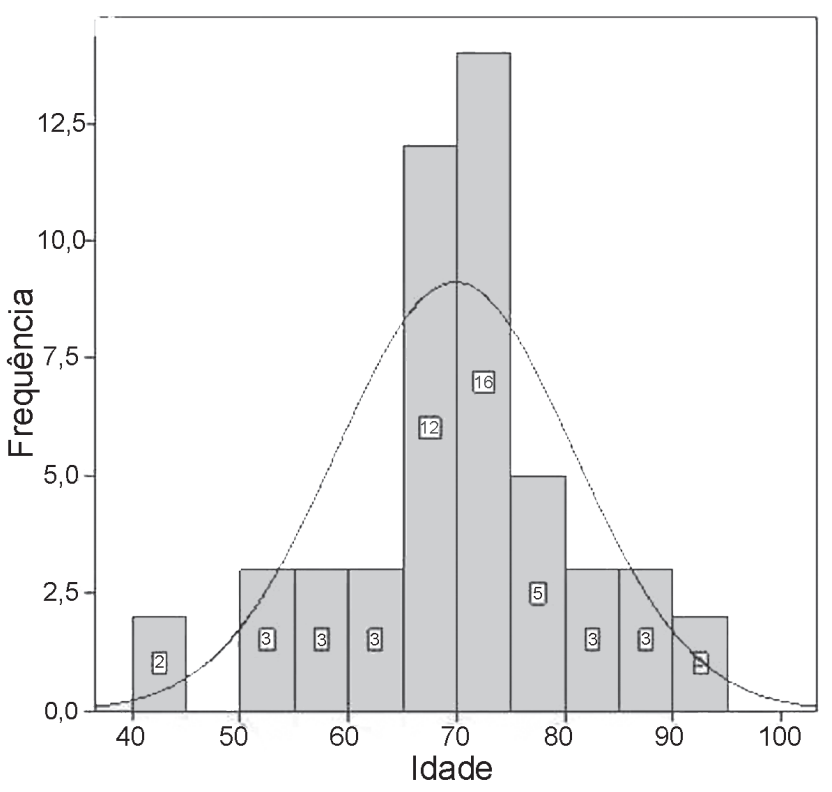

Grafico 1. Observou-se normalidade na distribuição quanto à idade em pacientes com LLC-B. Teste de Kolmogorov-Smirnov com ValorP de 0.942, confirmando a hipótese de distribuição normal.

Tabela 2. Estatística Descritiva dos Dados

\begin{tabular}{lc}
\hline & Amostra $(\mathrm{n}=50)$ \\
\hline Idade (anos) & $69,84 \pm 10,95$ \\
Hemoglobina $(\mathrm{g} / \mathrm{dL})$ & $12,26 \pm 2,25$ \\
Leucócitos $(/ \mathrm{LL}$ & $78.626,40 \pm 87.464,49$ \\
Linfócitos $(\%)$ & $83,55 \pm 12,99$ \\
Plaquetas $(/ \mu \mathrm{L})$ & $190.079 \pm 104856$ \\
$\mathrm{LDH}(\mathrm{U} / \mathrm{L})$ & $419,02 \pm 293,45$ \\
\hline
\end{tabular}

Os resultados foram reportados em valores de Média \pm Desviopadrão

a classificação da LLC segundo Rai et al (Tabela 1). Decidiuse por incluir também no estágio Rai 0 os pacientes com linfócitos abaixo de $15.000 / \mu \mathrm{L}$, mas diagnosticados, independentemente, como LLC-B positivos e não classificáveis quanto aos demais estágios clínicos.

Assim, foram obtidos os seguintes índices percentuais: Rai 0 - 48\% (n=24), Rai I - 6\% (n=3), Rai II - 18\% (n=9), Rai III - 24\% ( $\mathrm{n}=12)$ e Rai IV - 4\% (n=2) (Gráfico 2).

Os valores de CD38 disponíveis em 25 dos 50 prontuários, mostraram-se conforme o Gráfico 3 , com apenas 4 pacientes $(16 \%) \mathrm{CD} 38+\mathrm{e} 21(84 \%) \mathrm{CD} 38-$. Quanto à LDH, o valor médio encontrado foi de $419,02( \pm 293,45) \mathrm{U} / \mathrm{L}$, exceto por 4 pacientes $(8 \%)$, os demais $(92 \%)$ apresentaram níveis séricos acima do limite superior de referência (200 U/L).

Além das médias das variáveis calculadas para este estudo podem ser vistas na Tabela 2, enquanto os valores individuais quanto às variáveis numéricas puderam ser visualizados nos gráficos de dispersão. Porém, dentre as combinações de comparação possíveis para as variáveis, 


\section{Classific ação Rai}

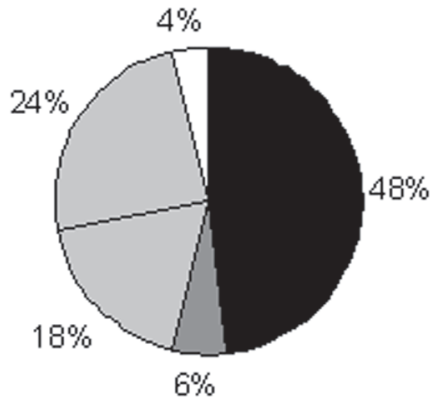

Rai $0 \square$ Rai I Rai II $\square$ Rai III $\square$ Rai IV

Gráfico 2. Estadiamento clínico, segundo Rai et al, de 50 pacientes com LLC-B: \&nbsp;Rai \&nbsp;0 - 48\% ( $n=24)$, Rai I - 6\% $(n=3)$, Rai II - 18\% ( $n=9)$, Rai III - 24\% ( $n=12)$ e Rai IV - 4\% ( $n=2)$. Níveis prognósticos: Rai 0 - inicial; Rai I e II - níveis intermediários; Rai III e IV - níveis avançados.

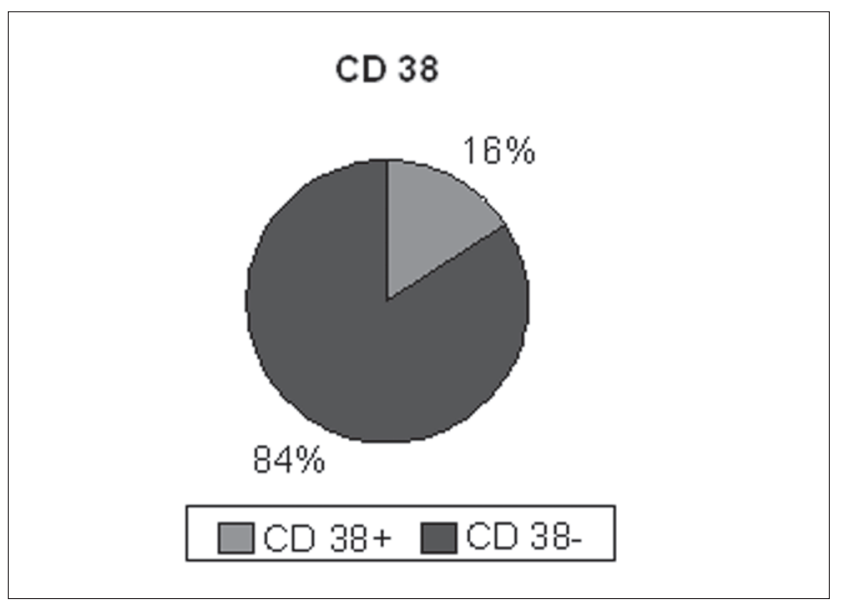

Gráfico 3. Perfil de imunofenotipagem quanto ao CD38 de 25 pacientes com LLC-B: $16 \%(n=4)$ positivos e $84 \%(n=21)$ negativos. A positividade é associada a um pior prognóstico, com evolução clínica e resposta ao tratamento desfavoráveis.

apenas para $\mathrm{LDH}(\mathrm{y})$ versus Leucócitos(x) (r=0.4962; $\left.\mathrm{y}=288.1+0.001 \mathrm{x} ; \mathrm{R}^{2}=0.246\right)$ e Leucócitos $(\mathrm{y})$ versus Linfó$\operatorname{citos}(\mathrm{x})\left(\mathrm{r}=0.1913 ; \mathrm{y}=447.82 ; \mathrm{R}^{2}=0.172\right)$ foi possível se propor modelos de regressão linear e exponencial, respectivamente, já que para as demais comparações, inclusive quanto às plaquetas, não foram encontrados coeficientes de determinação com valores suficientes para um modelo gráfico representativo ou com mínima incerteza quanto a previsões estatísticas. Quanto à última comparação (Leucócitos x Linfócitos), através de uma regressão estrutural, foi proposto também um modelo linear, com representatividade de $94.4 \%$ dos dados no modelo Linfócitos(y) versus Leucócitos(x) $\left(y=0.00225 x+0.00232(x-45000) ; R^{2}=0.944\right)$.
Foram comparadas entre si as médias de cada variável numérica quanto aos valores categóricos Rai e CD38, para se tentar atribuir alguma diferença nas suas distribuições dentre as possíveis categorias. Entretanto, ao contrário do que se esperava, não houve diferença significativa, exceto quanto à comparação entre plaquetas e estágios Rai.

\section{Discussão}

Nosso estudo evidenciou um predomínio menos acentuado, embora ainda significativo, nos homens, com $56 \%$ dos pacientes do sexo masculino e $44 \%$ do sexo feminino $(1,3: 1)$. Os dados na literatura quanto a isto são conflitantes. ${ }^{1}$

Quanto à idade, a grande maioria dos pacientes (74\%) mostrou-se acima dos 65 anos, em concordância com as estatísticas, ${ }^{1}$ as quais afirmam um aumento de até 20 vezes na incidência para pacientes acima dos 65 anos em comparação à faixa-etária inferior.

Atualmente, para o diagnóstico de LLC-B, não é especificada uma contagem mínima de linfócitos no sangue periférico. Especificações quanto à morfologia foram incluídas (antes não especificadas), devendo os linfócitos ser pequenos e maduros, sem nucléolo visível, e, caracteristicamente acompanhados de células de gumprecth. Além disso, a avaliação da medula óssea não é mais necessária, ao invés da uma contagem $\geq 30 \%$ de linfócitos sugerida anteriormente. ${ }^{7}$

Os demais critérios foram mantidos, como ao menos um dos marcadores de células B (CD19, CD20 ou CD23) e positividade quanto ao CD5 (na ausência de células T). Também é característica a negatividade para FMC7, CD22 (ou baixa expressão) e CD79b, com baixa densidade de Ig de superfície. Além disso, expressão monoclonal de cadeia $\kappa \mathrm{ou}$ $\gamma,<55 \%$ ou $<15.000 / \mu \mathrm{L}$ de células atípicas (pró-linfócitos) e cronicidade na duração da linfocitose, continuam necessárias e importantes no diagnóstico diferencial. ${ }^{7}$

Para os pacientes deste estudo, observou-se uma distribuição das contagens totais de linfócitos ao diagnóstico em três grandes faixas: $32 \%$, entre $10.000-25.000 / \mu \mathrm{L} ; 24 \%$ entre 25.000-50.000/ $\mu$ Le $26 \%$ entre 50.000-100.000/ $\mu \mathrm{L}$. Apenas $14 \%$ dos pacientes possuíam taxas de $100.000-300.000 / \mu \mathrm{L}$ e $4 \%$ acima disto.

A média percentual de linfócitos foi de 83,55\% ( $\pm 12,99 \%)$, que sobre a média leucocitária de 78.626,40 $( \pm 87.464,49)$ leucócitos $/ \mu \mathrm{L}$, gerou uma média de 65.692 linfócitos $/ \mu \mathrm{L}$, mais que o dobro do esperado em relação à média de contagem de linfócitos ao diagnóstico, em torno de $30.000 / \mu \mathrm{L},{ }^{3}$ com $52 \%$ dos pacientes acima de 40.000 linfócitos $/ \mu \mathrm{L}$.

Alguns autores, entretanto, afirmam haver uma diminuição de 8,6 para 3,7 anos de sobrevida para contagens, respectivamente de $20.000 / \mu \mathrm{L}$ e $40.000 / \mu \mathrm{L}^{3}$, em cuja categoria de pior prognóstico cairiam os $52 \%$ de pacientes deste estudo com linfócitos superiores a 40.000/ $\mu \mathrm{L}$. Mas é óbvia a necessidade de se levar em conta os demais fatores, em especial os novos marcadores de mutação $\operatorname{IgV}_{\mathrm{H}}$. 
A comparação de linfócitos e leucócitos através dos gráficos de dispersão só veio mesmo a confirmar estatisticamente a relação direta entre o aumento de leucócitos à custa de linfócitos, principalmente, como já esperado no diagnóstico da LLC-B.

Já contagens diminuídas de hemoglobina e plaquetas são tidas há décadas como sinônimos de estágio avançado na LLC, correspondendo aos estágios Rai III e IV, respectivamente, independentemente da presença ou ausência de linfonodopatias ou hepatoesplenomegalia. O diagnóstico, já nestes estágios, levaria a uma expectativa média de vida de apenas 19 meses para ambos os grupos, em comparação a mais de 150 meses no grupo Rai 0 ou 101 meses para o grupo Rai I. ${ }^{14}$ Durante os estágios mais avançados da doença, os pacientes podem desenvolver trombocitopenia devido à substituição de componentes normais da medula e/ou hiperesplenismo, podendo isto ocorrer, entretanto, em qualquer estágio pela produção de anticorpos antiplaquetários. ${ }^{17}$

Em torno de $15 \%$ de pacientes com LLC apresentam normalmente anemia normocítica normocrômica ao diagnóstico. ${ }^{17}$ Outro autor afirma que aproximadamente $30 \%$ dos pacientes podem ter, em determinados níveis, diminuição nas concentrações de hemoglobina ou plaquetas, mas que diminuições significativas $(\mathrm{Hb}<11 \mathrm{~g} / \mathrm{dL}$ e plaquetas $<100.000 / \mu \mathrm{L})$ estão presentes em apenas $15 \%$ dos mesmos. ${ }^{18}$

Os dados deste estudo demonstraram, entretanto, um percentual bem maior, com $32 \%(\mathrm{Hb}<11,5 \mathrm{~g} / \mathrm{dL})$ ou $26 \%(\mathrm{Hb}$ $<11,0 \mathrm{~g} / \mathrm{dL}$ ) de pacientes anêmicos já ao diagnóstico (apesar de apenas 2 pacientes com trombocitopenia inferior a 100.000 plaquetas $/ \mu \mathrm{L}$ ). Isto refletiu-se conseqüentemente em um percentual considerável de pacientes já diagnosticados no estágio III de Rai (alto risco), fato que, juntamente com a linfocitose já bastante acentuada $(>50.000 / \mu \mathrm{L}) \mathrm{em}$ mais da metade dos pacientes, corrobora também quanto à hipótese de falta de diagnóstico e tratamento precoce nesta população.

Baseados nos critérios originais propostos por Rai, realizou-se o estadiamento dos pacientes em estudo, obtendose as seguintes proporções para cada estágio: Rai 0 - 48\% $(n=24)$, Rai I - 6\% (n=3), Rai II - 18\% (n=9), Rai III - 24\% (n=12) e Rai IV - 4\% (n=2) (Gráfico 2).

Comparando-se com as proporções encontradas por Rai em 1975, observa-se, na população deste estudo, um aumento nos percentuais de pacientes tanto nos grupo de baixo risco ( $48 \%$ vs $31 \%$ ) como de alto risco ( $28 \%$ vs $8 \%$ ), e uma menor proporção no grupo de risco intermediário ( $24 \%$ vs $61 \%$ ). Além disso, caso o ponto de corte para trombocitopenia fosse aumentado de $100.000 / \mu \mathrm{L}$ para $150.000 / \mu \mathrm{L}$, ao invés de apenas 2 pacientes Rai IV, teriam sido classificados 22 pacientes (44\%) neste último estágio, os quais, somados aos pacientes então classificados como Rai III, dariam um total de 29 pacientes de alto risco ( $58 \%$ do total).

Além destes indicadores clínicos, dois outros marcadores foram avaliados neste estudo, quando disponíveis nos prontuários.
A enzima lactato-desidrogenase (LDH), embora atualmente de utilidade controversa, se considerada isoladamente ${ }^{8}$ tem sido associada a mau prognóstico, junto a outros marcadores sorológicos, embora faltem estudos a respeito, inclusive sobre os pontos de corte e metodologias empregadas. ${ }^{19}$ Contudo, foram coletados os dados dos 50 pacientes quanto ao nível desta enzima. Como mostrado nos gráficos. A LDH não mostrou diferenças estatisticamente significativas em suas médias ou medianas quanto aos diferentes estágios Rai (Gráfico 4), nem tampouco apresentou comportamento linear frente às concentrações de hemoglobina, plaquetas ou linfócitos. Entretanto apresentou um coeficiente linear significativo $(\mathrm{r}=0.4962)$ frente a leucócitos totais, e por regressão linear via teste $\mathrm{F}$, propôs-se o modelo ajustado $\mathrm{y}=$ $288.1+0.001 \mathrm{x}(\mathrm{y}=\mathrm{LDH}$ e $\mathrm{x}=$ leucócitos $)$, com coeficiente de determinação $\left(\mathrm{R}^{2}\right)$ de 0.246 (24,6\% dos dados representados na reta. Apesar desta relação diretamente proporcional entre LDH e leucócitos totais, a não diferença de suas médias dentre as categorias Rai, demonstrou a não correlação deste marcador com o estadiamento clínico.

Além disso, não houve diferença entre as médias de LDH e as duas categorias de CD38 (positivo ou negativo), sendo comprovada estatisticamente a igualdade entre as duas categorias através de dois testes estatísticos, com Valores-P respectivamente de 0.604 e 0.497 (Gráfico 5).

Foram observadas também discrepâncias quanto ao esperado, com pacientes apresentando linfócitos acima de $300.000 / \mu \mathrm{L}$, porém com nível de $\mathrm{LDH}$ equivalente à média observada para outros pacientes em Rai 0, inclusive. Este marcador, de fato, não parece ser confiável nem mesmo na predição de atividade tumoral, presente geralmente nas altas contagens de linfócitos.

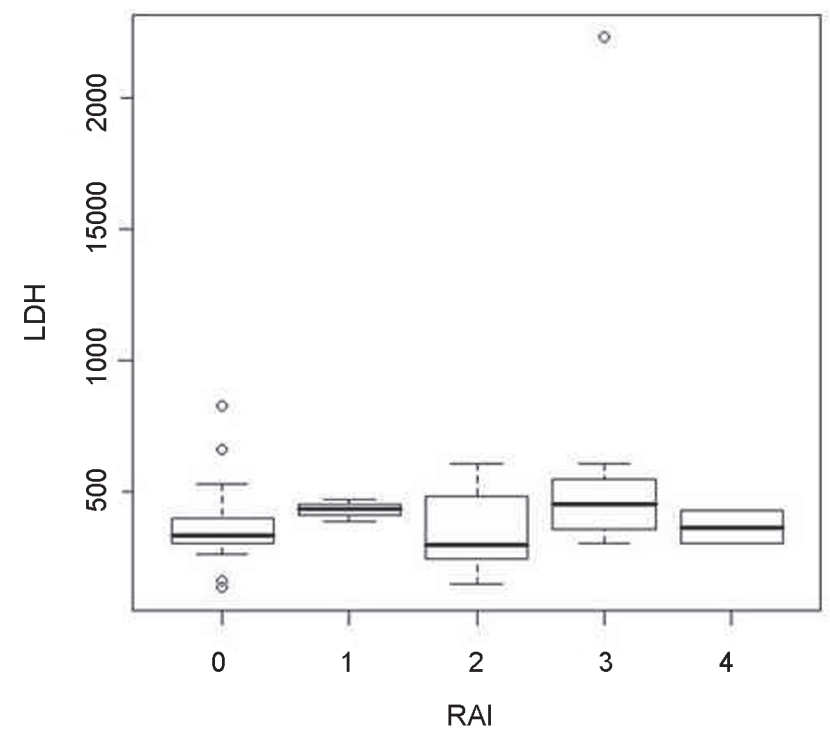

Gráfico 4. Gráfico de comparação entre as médias de LDH e categorias RAI dos 50 pacientes com LLC-B. A hipótese de igualdade foi confirmada através dos testes Kruskal-Wallis e Teste da Mediana, com valores-P de 0.092 e 0.075 , respectivamente. 


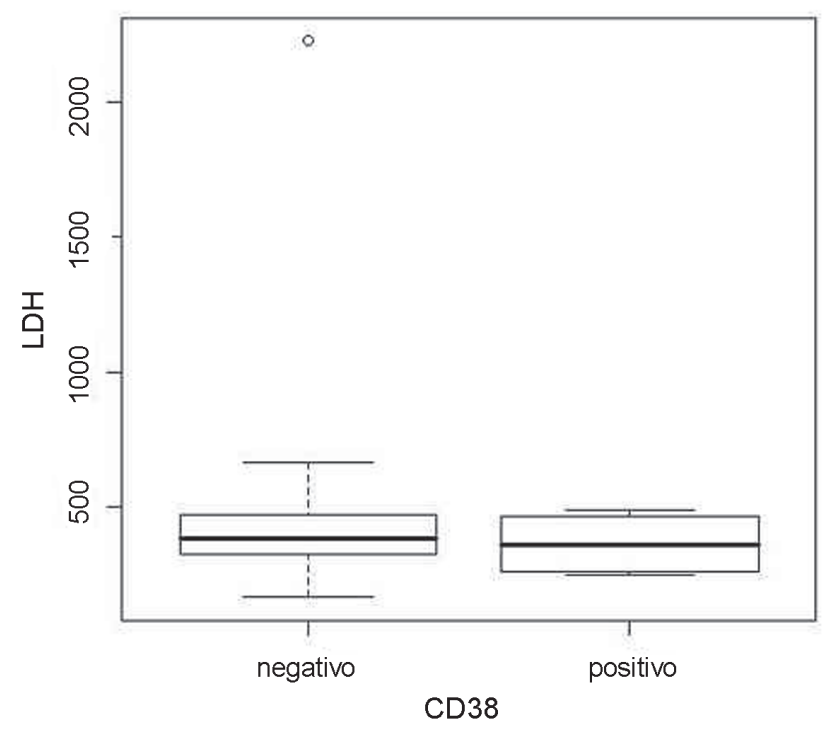

Gráfico 5. Gráfico de comparação entre as médias de LDH e categorias CD38 de 25 pacientes com LLC-B. A hipótese de igualdade foi confirmada através dos testes Mann-Whitney e KolmogorovSmirnov, com valores-P de 0.604 e 0.497 , respectivamente.

Já a fenotipagem para $\mathrm{CD} 38$, um dos marcadores mais importantes atualmente e associado à não-mutação $\operatorname{IgV}_{\mathrm{H}}$, de mau prognóstico, foi também coletado de 25 prontuários, nos quais estava disponível.

O Gráfico 3 mostra os resultados obtidos para o CD38. Dentre os 25 pacientes com fenotipagem realizada, 4 (16\%) apenas apresentaram $\mathrm{CD} 38+$ (mau prognóstico), enquanto 21 (84\%) apresentaram CD38- (bom prognóstico).

Embora em vários estudos os valores positivos quanto a este marcador estejam associados a um perfil de pior prognóstico, o qual incluiria também estágios clínicos mais avançados da LLC-B, curiosamente, dos quatro pacientes positivos, dois foram classificados como Rai III, um como Rai II e o outro como Rai 0, observando-se portanto positividade de CD38 tanto no grupo clínico de baixo risco, como no intermediário como no de nível avançado. Caso o ponto de corte para trombocitopenia fosse aumentado para $150.000 / \mathrm{mm}^{3}$, entretanto, todos os quatro pacientes teriam entrado clinicamente para o grupo de alto risco (três Rai IV e um Rai III), o que nos pareceria mais coerente quanto à positividade do CD38 (tido como um dos marcadores mais específicos hoje em dia).

Ainda, dentre os 25 com CD38 disponível, 21 foram reconhecidos como CD38- e, destes, 11 (52\%) eram de baixo risco clínico, cinco (24\%) de risco intermediário e cinco (24\%) de alto risco.

Portanto, quanto ao $\mathrm{CD} 38$, ao contrário do que se esperava, não houve concordância com grande parte dos pacientes quanto ao prognóstico clínico, pois dentre os casos CD 38 + haviam apenas $25 \%$ (um paciente) também classificados clinicamente como de estágio avançado, enquanto dos casos CD38- apenas 52\% foram classificados como de baixo risco, demonstrando valores preditivos positivos e negativos bastante baixos quando tomado o CD38 ou a classificação clínica de Rai como padrão mais confiável quanto ao melhor prognóstico.

\section{Conclusão}

Traçou-se o perfil dos cinquenta pacientes diagnosticados com LLC-B, realizando-se o estadiamento segundo Rai e comparando-se as variáveis inclusas neste estadiamento entre si e entre elas e os marcadores LDH e CD38.

O estadiamento clínico, apesar de suas limitações em relação aos marcadores mais recentes, pareceu-nos ainda útil quanto ao alerta que fez em relação a um número bastante significativo de pacientes já diagnosticados em estágio avançado, com $32 \%$ do total de pacientes anêmicos $(<11,5 \mathrm{~g} /$ $\mathrm{dL}), 44 \%$ trombocitopênicos $(<150.000$ plaquetas $/ \mu \mathrm{L}), 48 \%$ com linfadenopatia e $32 \%$ com sinais de hepatoesplenomegalia. A classificação Rai foi, portanto, de $48 \%$ de pacientes em Rai 0; 6\% em Rai I, 18\% em Rai II, 24\% em Rai III e 4\% em Rai IV; logo, 48\% em estágio inicial, 24\% em estágio intermediário e $28 \%$ em estágio já avançado ao diagnóstico, com um aumento considerável tanto no estágio inicial quanto avançado em relação a outros estudos baseados nesta classificação clínica.

Estatisticamente, observou-se uma normalidade de distribuição das variáveis numéricas dentro da população, exceto para leucócitos totais e linfócitos. Na comparação estatística entre variáveis, através de gráficos de dispersão, apenas notou-se diferença significativa entre leucócitos totais e LDH, e entre leucócitos totais e linfócitos, a partir das quais propôs-se modelos lineares ajustados por regressões lineares.

Também, nos gráficos de comparação entre as médias das variáveis numéricas (inclusive LDH) com as categorias Rai ou de CD38, ao contrário do que se esperava, não se observou diferença estatísticamente significante, o que mostrou a incoerência entre estes marcadores e o estadiamento clínico, e entre eles próprios (CD38 e LDH), inclusive quanto a discrepâncias pontuais em pacientes isoladamente.

Os resultados portanto convergem para a necessidade de estudos em populações mais amplas, em que outros marcadores tumorais, como a beta-2-microglobulina, ZAP70 e outros, ou mesmo o estudo citogenético e do estado mutacional de $\operatorname{IgV}_{\mathrm{H}}$ sejam cuidadosamente avaliados, e o conjunto seja tomado como referência no julgamento dos valores prognósticos de cada marcador separadamente ou mesmo da ainda validade das classificações clínicas quanto à maior especificidade dos marcadores tumorais mais recentes.

Também se faz imprescindível um aumento na educação da população quanto ao direito e dever pela busca de um serviço de saúde de qualidade, inclusive em suas localida- 
des próximas, e de uma atenção maior à saúde preventiva, através de ações multidisciplinares, para o aumento no percentual de diagnósticos da LLC-B em estado inicial e diminuição dos pacientes que chegam aos grandes centros já em estágio avançado.

Investimentos maiores em remuneração e valorização profissional também são fundamentais para que os profissionais envolvidos sigam obtendo o que há de melhor em educação continuada e formação acadêmica quanto às tecnologias mais recentes, o que certamente traria resultados positivos consideráveis e essenciais na prática da clínica hematológica e rotinas laboratoriais.

\begin{abstract}
It has been given great importance to the advances in molecular biology and genetics in the establishment of new protocols, as well as to the discovery of new tumoral markers, useful to early diagnosis and treatment of various cancer diseases, inclusive $B$ cell Chronic Lymphocytic Leukemia (B-CLL). For evaluation of the actual significance of Rai clinical staging system in the prognosis of 50 B-CLL patients at a state hemocenter, as well as for comparison between the staging results with the serum values of LDH and CD38 at diagnosis, it was collected from patient promptuaries the data regarding the total lymphocyte and platelet counting, hemoglobin concentration, and also information about the presence or absence of linfadenopathy and/or organomegaly. When compared the staging results with those of other studies, it was noted a worrying situation considering the superior percentage of patients already classified as of high risk at diagnosis. This picture of apparent delay in the detection of CLL-B was to some extent attributed to the difficulty of access to specialized centers and/or delay in hematological evaluation. Additionally, it was observed some incoherence between $\mathrm{LDH}$ and CD38, and between these and the clinical classification system of Rai. The results suggest that this classification system may still be useful as a general comparative prospect of B-CLL between different populations, but also emphasize the requirement of specific prognostic models that consider, beyond the clinical data e the markers CD38 and $\mathrm{LDH}$, other more precise indicatives of IgVH mutational status for more accurate prognosis and therapeutics. Rev. Bras. Hematol. Hemoter. 2009;31(4):228-234.
\end{abstract}

Key words: Hematologic profile; chronic lymphocytic leukemia; prognosis.

\section{Referências Bibliográficas}

1. Yamamoto M, Figueiredo VLP. Epidemiologia da Leucemia Linfocítica e Leucemia Linfocítica Crônica Familiar. Revista Brasileira de Hematologia Clínica. 2005;27(4):229-232.

2. Nascimento MC, Yamamoto $\mathrm{M}$, Rodrigues $\mathrm{MM}$, et al. Chromosomal Abnormalities (FISH) and their Relation with Clinical Stage, CD38 and ZAP-70. Rev Bras. Hematol. Hemoter. 2006;28 (1):5-10.

3. Greer JP, Foerster J, Lukens JN, et al - Wintrobe's Clinical Hematology, 11. ed. americana. Philadelphia (USA): Lippincott Williams \& Wilkins, 2003, 2800 p.
4. Ferrarini M, Chiorazzi N. Recent advances in the molecular biology and immunobiology of chronic lymphocytic leukemia. Semin Hematol. 2004;41(3):207-23.

5. Seiler T, Döhner H, Stilgenbauer S. Risk stratification in chronic lymphocytic leukemia. Semin Oncol. 2006;33(2):186-94.

6. Vasconcelos Y. Marcadores de Prognóstico na Leucemia Linfocítica Crônica. Rev. Bras. Hematol. Hemoter. 2005;27(4):253-6.

7. Yee KW, O'Brien SM. Chronic lymphocytic leukemia: diagnosis and treatment. Mayo Clin Proc. 2006;81(8):1105-29.

8. Montillo M, Hamblin T, Hallek M, Montserrat E, Morra E. Chronic lymphocytic leukemia: novel prognostic factors and their relevance for risk-adapted therapeutic strategies. Haematologica. 2005; 90 (3):391-9.

9. Del Principe MI, Del Poeta G, Buccisano F, Maurillo L, Venditti A, Zucchetto A, et al. Clinical significance of ZAP-70 protein expression in B-cell chronic lymphocytic leukemia. Blood. 2006 Aug 1;108(3):853-61.

10. Montserrat E. New prognostic markers in CLL. Hematology Am Soc Hematol Educ Program. 2006:279-84.

11. Deaglio S, Vaisitti T, Aydin S, Ferrero E, Malavasi F. In-tandem insight from basic science combined with clinical research: CD38 as both marker and key component of the pathogenetic network underlying chronic lymphocytic leukemia. Blood. 2006;108(4): 1135-44.

12. Deaglio S, Vaisitti T, Aydin S, Bergui L, D'Arena G, Bonello L, et al. CD38 and ZAP-70 are functionally linked and mark CLL cells with high migratory potential. Blood. 2007;110(12):4012-21.

13. Deaglio S, Aydin S, Vaisitti T, Bergui L, Malavasi F. CD38 at the junction between prognostic marker and therapeutic target. Trends Mol Med. 2008;14(5):210-8.

14. Rai KR, Sawitsky A, Cronkite EP, Chanana AD, Levy RN, Pasternack BS. Clinical staging of chronic lymphocytic leukemia. 1975;46(2):219-34.

15. Binet JL, Lepoprier M, Dighiero G, Charron D, D'Athis P, Vaugier G, et al. A clinical staging system for chronic lymphocytic leukemia: prognostic significance. Cancer. 1977;40(2):855-64.

16. Hallek M. Chronic Lymphocytic Leukemia (CLL): First-Line Treatment. Hematology Am Soc Hematol Educ Program. 2005: 285-91.

17. Beutler E; Lichman MA, Coller BS, et al. Williams Hematology, $6^{\mathrm{a}}$ ed. americana. USA: McGraw-Hill Professional, 2000, 1941 p.

18. Hoffman R, Silberstein L, Benz EJ. Hematology: Basic Principles and Practice, $3^{\mathrm{a}}$ ed. Americana. USA: Churchill Livingstone, 2000, $2584 \mathrm{p}$.

19. Oscier D, Fegan C, Hillmen P, Illidge T, Johnson S, Maguire P, et al. Guidelines on the diagnosis and management of chronic lymphocytic leukaemia. Br J Haematol. 2004;125(3):294-317.

Avaliação: Editor e dois revisores externos Conflito de interesse: sem conflito de interesse.

Recebido: 05/09/2008

Aceito após modificações: 24/03/2009 